PROCEEDINGS OF THE

AMERICAN MATHEMATICAL SOCIETY

Volume 129, Number 6, Pages 1643-1645

S 0002-9939(00)05747-6

Article electronically published on October 31, 2000

\title{
IRREDUCIBLE RESTRICTION AND ZEROS OF CHARACTERS
}

\author{
GABRIEL NAVARRO
}

(Communicated by Stephen D. Smith)

\begin{abstract}
Let $G$ be a finite group, let $N$ be normal in $G$ and suppose that $\chi$ is an irreducible complex character of $G$. Then $\chi_{N}$ is not irreducible if and only if $\chi$ vanishes on some coset of $N$ in $G$.
\end{abstract}

\section{INTRODUCTION}

Let $N \triangleleft G$, where $G$ is an arbitrary finite group, and let $\chi \in \operatorname{Irr}(G)$ be an irreducible complex character of $G$. In this note we give a characterization of when the restricted character $\chi_{N}$ is irreducible which at the same time extends Burnside's theorem on zeros of characters.

Theorem A. Let $G$ be a finite group and let $N \triangleleft G$. Let $\chi \in \operatorname{Irr}(G)$. Then $\chi_{N}$ is not irreducible if and only if $\chi$ vanishes on some coset $N x$ of $N$ in $G$.

When $N=1$ (or more generally if $N$ is abelian) Theorem A is Burnside's theorem on zeros. We mention some easy consequences of Theorem A which again extend Burnside's theorem.

Corollary B. Let $N \triangleleft G$ with $G / N$ a $\pi$-group and let $\chi \in \operatorname{Irr}(G)$. If $\chi$ is nonzero on the $\pi$-elements of $G$, then $\chi_{N}$ is irreducible.

Corollary C. Let $N \triangleleft G$ and let $H \subseteq G$ be such that $G=H N$. Let $\chi \in \operatorname{Irr}(G)$. If $\chi(h) \neq 0$ for $h \in H$, then $\chi_{N}$ is irreducible.

I thank M. Isaacs for useful discussions on this note.

\section{Proofs}

Our proof of Theorem A is another application of the theory of character triple isomorphisms. The reader is referred to [1] for its definition and main properties.

Proof of Theorem A. Suppose first that $\chi_{N}$ is irreducible. Let $x \in G$. By Lemma (8.14) of [1, we have that

$$
\sum_{g \in N x}|\chi(g)|^{2}=|N|
$$

and therefore $\chi$ cannot be zero on the coset $N x$.

Received by the editors September 28, 1999.

2000 Mathematics Subject Classification. Primary $20 \mathrm{C} 15$.

The author's research was partially supported by DGICYT.

(C)2000 American Mathematical Society 
Suppose now that $\chi_{N}$ is not irreducible. We want to find $x \in G$ such that $\chi(n x)=0$ for all $n \in N$. Let $\theta \in \operatorname{Irr}(N)$ be an irreducible constituent of $\chi_{N}$. Let $T$ be the stabilizer of $\theta$ in $G$ and by the Clifford correspondence (Theorem (6.11) of [1]), let $\psi \in \operatorname{Irr}(T)$ be such that $\chi=\psi^{G}$. Assume that $T<G$. Then

$$
\bigcup_{g \in G} T^{g} \subset G
$$

and we let $x \in G$ lie in no $G$-conjugate of $T$. Since $N$ is contained in every $G$ conjugate of $T$, it follows that for each element $n \in N$, the element $n x$ is contained in no $G$-conjugate of $T$. It follows that $\chi=\psi^{G}$ vanishes on the entire coset $N x$, by the character induction formula.

We can now assume that $T=G$. Hence $\chi_{N}$ is a multiple of $\theta$. By Theorem (11.28) of [1], let $\left(G^{*}, M, \nu\right)$ be a character triple isomorphic to $(G, N, \theta)$ with $M \subseteq \mathbf{Z}\left(G^{*}\right)$. Let $\chi^{*} \in \operatorname{Irr}\left(G^{*}\right)$ correspond to $\chi$. Let us denote by ${ }^{*}$ the group isomorphism $G / N \rightarrow G^{*} / M$ associated to the character triple isomorphism.

By Lemma (11.24) of [1, we have that

$$
e=\frac{\chi(1)}{\theta(1)}=\frac{\chi^{*}(1)}{\nu(1)}
$$

Since $\chi_{N}$ reduces, $e>1$ and we conclude that $\chi^{*}$ is nonlinear. By Burnside's theorem (Theorem (3.15) of [1]), there exists $y \in G^{*}$ such that $\chi^{*}(y)=0$. Now, let $V=\langle M, y\rangle$ and let $N \subseteq U \subseteq G$, where $(U / N)^{*}=V / N$. Since $U / N \cong V / M$ is cyclic, we see that $\theta$ has an extension $\varphi \in \operatorname{Irr}(U)$, by Corollary (11.22) of [1. By Gallagher's theorem (Corollary (6.17) of [1]), notice that we may write

$$
\chi_{U}=\psi \varphi
$$

for some character $\psi$ of $U / N$. Let us denote by $\psi^{*}$ the character of $V / N$ satisfying

$$
\psi^{*}\left((w N)^{*}\right)=\psi(w N)
$$

for $w \in U$. By Definition (11.23.d) of [1], we have that

$$
\chi_{V}^{*}=\psi^{*} \varphi^{*},
$$

where $\varphi^{*}$ is the extension of $\nu$ corresponding to $\varphi$ under the character triple isomorphism. Since $\nu$ is linear, we have that $\varphi^{*}$ is also linear.

Now, let the coset $N x \subseteq U$ correspond to $M y$ under the character triple isomorphism. Note that we can write $\psi(x)=\psi(N x)=\psi^{*}(M y)=\psi^{*}(y)$. Now

$$
0=\chi^{*}(y)=\psi^{*}(y) \varphi^{*}(y),
$$

and the second factor is nonzero because $\varphi^{*}$ is linear. Thus $\psi^{*}(y)=0$ and hence $\psi(x)=0$. It follows that

$$
\chi(x)=\psi(x) \varphi(x)=0 .
$$

Since $x$ was an arbitrary element of the coset $N x$, the result follows.

Proof of Corollary B. Suppose that $\chi_{N}$ is not irreducible. Then there exists a coset $N x$ of $N$ in $G$ on which $\chi$ is zero. Now $N x=N x_{\pi}$ contains the $\pi$-element $x_{\pi}$, and this contradicts the hypothesis.

Proof of Corollary C. If $\chi_{N}$ is not irreducible, there exists a coset $N x$ of $N$ in $G$ on which $\chi$ is zero. Now $N x$ contains some $h \in H$, and this contradicts the hypothesis. 


\section{REFERENCES}

[1] M. Isaacs, Character Theory of Finite Groups, New York, Dover, 1994.

Departament d’Àlgebra, Universitat de València, 461100 Burjassot, València, Spain E-mail address: gabriel@uv.es

Current address: Department of Mathematics, University of Wisconsin, Madison, Wisconsin 53706

E-mail address: navarro@math.wisc.edu 